ARTICLE / INVESTIGACIÓN

\title{
Identification of novel-vector control target proteins of Aedes sp.: A Systems Network Biology Approach
}

${ }^{3}$ Centre for Systems Biology and Bioinformatics, UIEAST, Panjab University, Chandigarh, India.

Corresponding author: tammanna@pu.ac.in

\begin{abstract}
Aedes is an important vector for various viruses that cause dengue, chikungunya and zika, which affect human health globally. Due to regular outbreaks of these diseases worldwide, there is a need to identify essential vector proteins that are critical for the survival of the vector, which may be targeted to control the spread of vector-borne disease (VBD). In silico computational methods involving comparative proteomics, analysis of orthologous proteins common amongst members of Aedes genus and protein-protein interaction (PPI) pathway were used to identify essential proteins that could act as novel therapeutic candidates. Twenty-three conserved proteins between A. aegypti and A. albopictus were identified from a BLASTP search with an e-value threshold of 0.005, and their PPI networks were constructed in the STRING database. The merged network was analyzed using various Cytoscape plugins viz. ClusterONE, Cytohubba and MCODE. Thirty-one hub proteins were identified from the system's network biology analysis, and detailed data and literature mining were carried out. Twelve novel vector-control target proteins of $A$. aegypti, having no human homologs, were determined in the present study that can effectively act as potential therapeutic candidates for drug design and vaccine development.
\end{abstract}

Key words: Vector-borne disease (VBD), Therapeutic candidates, Network Systems Biology, Protein-protein interactions (PPI), Computational biology.

\section{Introduction}

Vector-borne diseases (VBD) are significant contributors to the global disease burden and account for more than $17 \%$ of all infectious diseases, causing more than 700000 deaths annually ${ }^{1}$. An important taxon of arthropods that affects human health globally by acting as a vector of arthropod-borne viruses are mosquitoes belonging to the Aedes genus. It's been over five decades that Aedes-borne diseases, such as dengue, Zika, chikungunya, and yellow fever, have emerged and re-emerged globally ${ }^{2,3}$. The global population at risk is expanding in concert with changes in the distribution of two key vectors: Aedes aegypti and Aedes albopictus. Their desiccation-resistant eggs facilitate the invasion of Aedes into new areas, and the distribution of these species is largely driven by both human movement and the presence of a suitable climate ${ }^{4}$.

Dengue virus has been reported to cause about $390 \mathrm{mi}-$ Ulion human infections per year; the chikungunya virus spread worldwide in the early 2000s, with recent reports of the spread of Zika virus, while yellow fever has resurged in Africa and America. In most cases, vector control is the only means to cope with various foreign diseases.

The feeding preference of Aedes is only mammalian species, including birds, mammals and humans, which increases during the multiplication phase of their life cycle. The transmission of viruses from the mosquitoes to the organisms sharing the same niche is due to the bite of the infected vector, which releases the virus into the body of the recipient organism. Aedes aegypti was earlier found in forested territories, utilizing tree openings as natural surroundings as their habi- tat $^{5}$. Now, they have transformed and evolved along with the increasing urbanization and have found their habitat in leftover jars, tires, broken water-filled buckets and even in the tanks or ponds having stagnant water. They usually prefer areas where they can easily find their host and feed on them eventually ${ }^{6}$.

Another member of the Aedes genus is now listed as one of the top 100 invasive species by the Invasive Species Specialist Group ${ }^{7}$ on account of being a suitable vector for causing human diseases chikungunya virus, dengue virus, and dirofilariasis is $A$. albopictus. Experimental evidence has shown that its preferred host is humans, but being an opportunistic feeder, it can depend upon other hosts such as domestic and wild animals, birds, reptiles, and amphibians for its food cycle $e^{8,9}$

Vector-borne diseases (VBD) are a worldwide concern and a significant cause of human morbidity and mortality, causing immeasurable suffering and impeding economic development. To protect humans from these destructive diseases, controlling insect vectors is often the most suitable and sometimes the only option available. Many control strategies have been designed and followed since centuries ago, but despite such efforts and programs, mosquito-borne diseases are prospering throughout the world ${ }^{10}$.

The amalgamation of "omics" data with computational biology tools in network systems biology offers the advantage of speedy identification of critical vector proteins necessary for the survival of the vectors, which may play an essential role in controlling vector populations. In silico methods such as comparative and subtractive genomics can be effectively be used

Citation: Sahrawat TR, Talwar D and Patial R. Identification of novel-vector control target proteins of Aedes sp.: A Systems Network Biology Approach. Revis Bionatura 2022;7(1). 12. http://dx.doi.org/10.21931/RB/2022.07.01.12

Received: 2 August 2021 / Accepted: 18 November 2021 / Published: 15 February 2022

Publisher's Note: Bionatura stays neutral with regard to jurisdictional claims in published maps and institutional affiliations. 
to identify proteins/genes of vectors that are non-homologous to their human hosts. These proteins/genes may then be targeted using drugs and vaccines by inhibiting critical vector pathways and preventing host-seeking and feeding behaviors.

The present study was undertaken to identify critical vector-control target proteins for the Aedes genus using a network systems biology approach. Such an approach offers the opportunity to ascertain potential targets for drug development and vaccine design that would facilitate the production of novel therapeutics specific against the disease-carrying vectors.

\section{Materials and methods}

\section{Proteome data mining}

The UniProt KB database (https://www.uniprot.org) ${ }^{11}$ was used to extract proteins present in vector Aedes aegypti and for functional annotation of the essential proteins along with eggNOG (http://eggnog5.embl.de) ${ }^{12}$.

\section{BLAST}

Basic alignment search too ${ }^{13}$ was used to identify non-human homologs proteins of Aedes aegypti. This was followed by the identification of conserved essential proteins amongst two central disease-causing species of the Aedes genus, namely Aedes aegypti and Aedes albopictus.

\section{Vector specific pathway analysis using KEGG}

Complete form (KEGG) ${ }^{14}$ was used to ascertain the metabolic pathways of non-homologous essential proteins that were identified in the previous step.

\section{Sub-cellular location prediction}

To predict the subcellular location of the essential proteins, a consensus was drawn from the results obtained from UniProt ${ }^{11}$, Cello ${ }^{15}$, I-loc animal ${ }^{16}$, SherLoc $2^{17}$ and Wolf PSORT ${ }^{18}$.

\section{Host-vector protein-protein interactions}

\section{STRING}

(Search Tool for the Retrieval of Interacting Genes/Proteins) (https://string-db.org/) (version 11.0) database was used to build protein-protein interaction networks of essential proteins of Aedes aegypti and their interacting partners. The networks obtained were analyzed using Cytoscape (version 3.8.0) and its various plugins such as ClusterONE, CytoHubba, MCODE ${ }^{19}$.

\section{ClusterONE}

(http://apps.cytoscape.org/apps/clusterone). ClusterONE (Clustering with Overlapping Neighborhood Expansion) was used to identify highly connected regions in the form of clusters that were based on weighted graphs with associated confidence values ${ }^{20}$

\section{CytoHubba}

(http://apps.cytoscape.org/apps/cytohubba). CytoHubba with the scoring method of maximal clique centrality (MCC) was used to rank hub genes ${ }^{21}$.

\section{MCODE}

(http://apps.cytoscape.org/apps/mcode). MCODE (Molecular Complex Detection) was used to find highly connected regions called clusters based solely on connectivity data ${ }^{22}$.

\section{Results}

\section{Proteome data mining for identification of non-human homologs}

UniProt KB was used to retrieve proteins present in $\mathrm{Ae}$ des aegypti. To increase reliability and reduce redundancy in data mining, only the reviewed 253 protein data entries were selected. All the 253 protein sequences of $A$. aegypti were subjected to a BLASTP search against the host proteome of Homo sapiens with an e-value threshold of $0.005^{23}$. A total of 24 proteins of $A$. aegypti that showed "no significant similarity" to humans were shortlisted as non-human homologs (Table 1). After that, these proteins were analyzed using computational biology approaches to identify essential proteins of Aedes genus that may act as potential drug and vaccine targets.

\section{Identification of essential proteins in Aedes aegypti}

The presence of orthologous proteins between two or more species indicates their essentiality for the survival of the genus. Orthologous proteins are known to retain equivalent functions in different organisms ${ }^{24}$. Due to the absence of any information about the essential proteins/genes of $A$. aegypti in DEG (Database of Essential Genes), various bioinformatics tools were used to identify the orthologues. Twenty-four proteins of $A$. aegypti (Table 1 ) were subjected to a manual BLASTP search with e-value threshold of 0.005 , to identify conserved proteins against proteome of another Aedes genus member i.e A. albopictus. Unique orthologues proteins were selected manually using a cut-off $\geq 50 \%$ identity between $A$. aegypti and A. albopictus. Twenty-three proteins (except TKSIA_AEDAE) were identified as conserved essential proteins common between the Aedes species. These conserved proteins may be critical for the survival and pathogenicity of members of Aedes genus (Table 1).

\section{Vector specific pathway analysis and sub-localization prediction}

The 23 proteins identified as conserved essential proteins were then categorized as "pathway dependent" or "pathway independent" using UniProt KB GO and KEGG pathway database, and their subcellular location was determined by drawing consensus amongst results obtained from UniProt, Cello, I-loc animal, SherLoc2 and Wolf PSORT. Out of the 23 proteins, 20 were found to be pathway independent, and 3 proteins, namely DEFA_AEDAE, DEFB_AEDAE and DEFC_AEDAE were found pathway dependent, involved in innate immunity ${ }^{25}$. All these 23 proteins of $A$. aegypti that were absent in human hosts were further explored using a network systems biology approach to identify critical vector target proteins that can act as drug and vaccine targets to curb the spread of VBDs.

\section{Host-vector protein-protein interactions}

\section{Construction of gene interaction network}

The interaction network for the 23 essential proteins of Aedes aegypti was constructed using the STRING database. On analysis, it was observed that HPEP_AEDAE and BURS AEDAE were absent, while DEFA_AEDAE and DEFB_AEDAE were represented as the same protein in STRING results for Aedes sp. Therefore the resulting PPI network had 20 proteins that showed no significant interactions amongst themselves since these proteins were involved in different pathways of survival and pathogenesis of the vector (Figure 1). Three 


\begin{tabular}{|l|l|l|l|l|l|}
\hline \multicolumn{7}{|c|}{ Table 1. Essential proteins common in both Aedes aegypti and Aedes albopictus } \\
\hline $\mathbf{1}$ & COMM2_AEDAE & 9 & ATP8_AEDAE & 17 & DEFC_AEDAE \\
\hline $\mathbf{2}$ & V15A1_AEDAE & 10 & SNPF_AEDAE & 18 & CECA_AEDAE \\
\hline $\mathbf{3}$ & OBP45_AEDAE & 11 & V15A3_AEDAE & 19 & VIT1_AEDAE \\
\hline $\mathbf{4}$ & NPF_AEDAE & 12 & PBAN_AEDAE & 20 & DEFB_AEDAE \\
\hline $\mathbf{5}$ & LCK_AEDAE & 13 & DEFA_AEDAE & 21 & ALL3_AEDAE \\
\hline $\mathbf{6}$ & ALL2_AEDAE & 14 & OR4_AEDA & 22 & SHAKB_AEDAE \\
\hline $\mathbf{7}$ & CORZ_AEDAE & 15 & V15A2_AEDAE & 23 & HPEP_AEDAE \\
\hline $\mathbf{8}$ & BURS_AEDAE & 16 & ORCO_AEDAE & 24 & TKSIA_AEDAE* \\
\hline * rejected because it was not common between the Aedes aegypti and A. albopictus species \\
\hline
\end{tabular}

Table 1. Essential proteins common in both Aedes aegypti and Aedes albopictus.

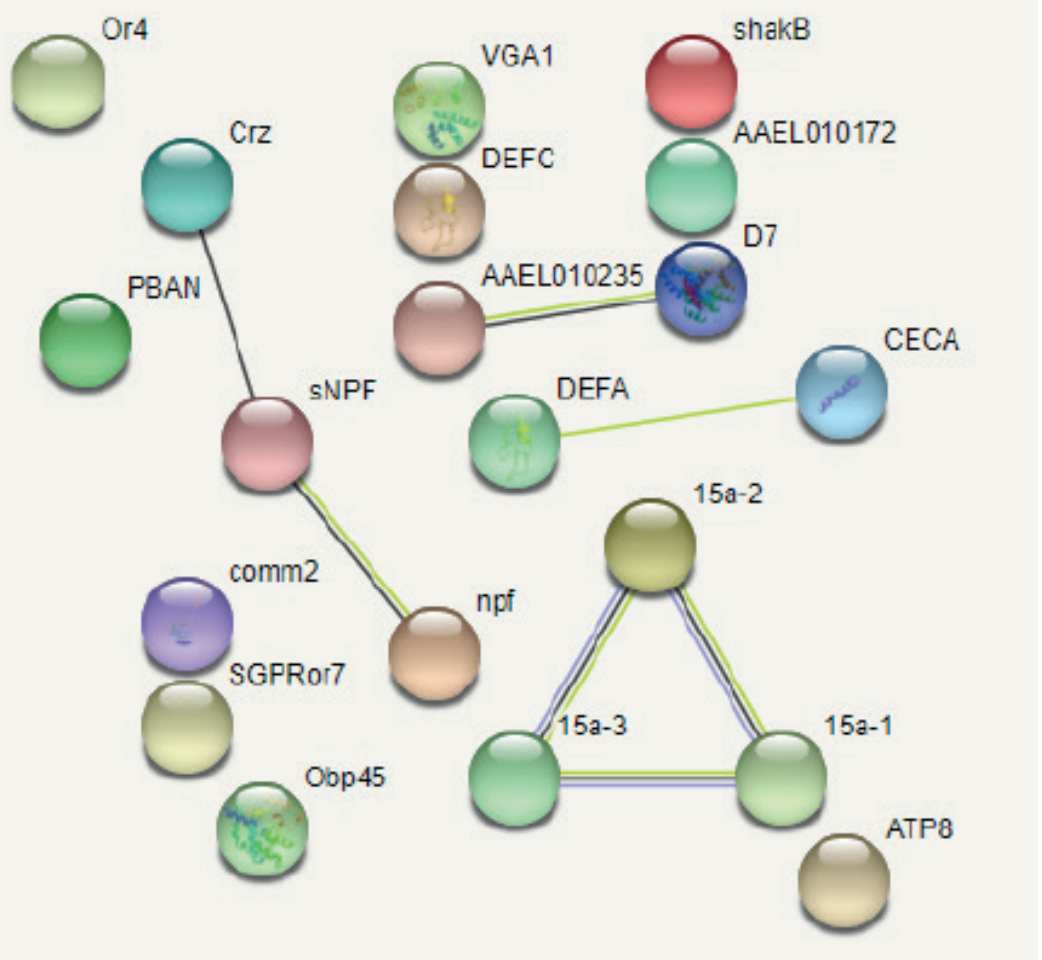

Figure 1. Intra-species interactions amongst Aedes proteins constructed in the STRING database.

PPIs were observed, i.e., ALL2_AEDAE (D7) and ALL3_AEDAE (AAEL010235) that are salivary gland allergens and reported to be involved in blood-feeding of the human host ${ }^{26,27}$; CORZ AEDAE (crz) and NPF_AEDAE with SNPF_AEDAE, in which all are neuropeptides with CORZ being involved in heart contraction $^{28} ;$ V15A1_AEDAE (15a-1), V15A2_AEDAE (15a-2) and V15A3_AEDAE (15a-3) are proteins involved in oogenesis and therefore may act as an essential control point in the reproduction of Aedes mosquito ${ }^{29,30}$.

To better understand the interactions of the essential proteins with other proteins of $A$. aegypti, interaction networks of each of the 20 proteins were built individually in STRING and were imported and merged into a single network using Cytoscape software. The resulting merged network contained 96 edges (representing genes/proteins) and 243 nodes (representing interactions between genes/proteins) (Figure 2). This network was further analyzed using various plugins of Cytoscape to identify essential proteins of $A$. aegypti.
On analysis of the merged network with Cytoscape plugin ClusterONE, color-coded nodes were obtained in which red and grey nodes represent highly significant genes and outliers, respectively (Figure 3 ). Eleven clusters were obtained that had a significant P-value $(<0.05)$ (Table 2$)$, but only three topranked clusters having the most significant number of nodes and edges were further studied.

Another Cytoscape plugin MCODE was used to find clusters or highly connected regions in the protein-protein interaction network in which node colors represent the node score and range from black to red (lowest to highest, respectively), while white indicates a score of zero (Figure 4). Eleven clusters were also obtained from MCODE, out of which the first three clusters were considered as significant based on the higher number of nodes and edges (Table 3 ).

To further validate the results of genes identified as essential using Cytoscape plugins- ClusterONE and MCODE another plugin CytoHubba was used to analyse the merged 

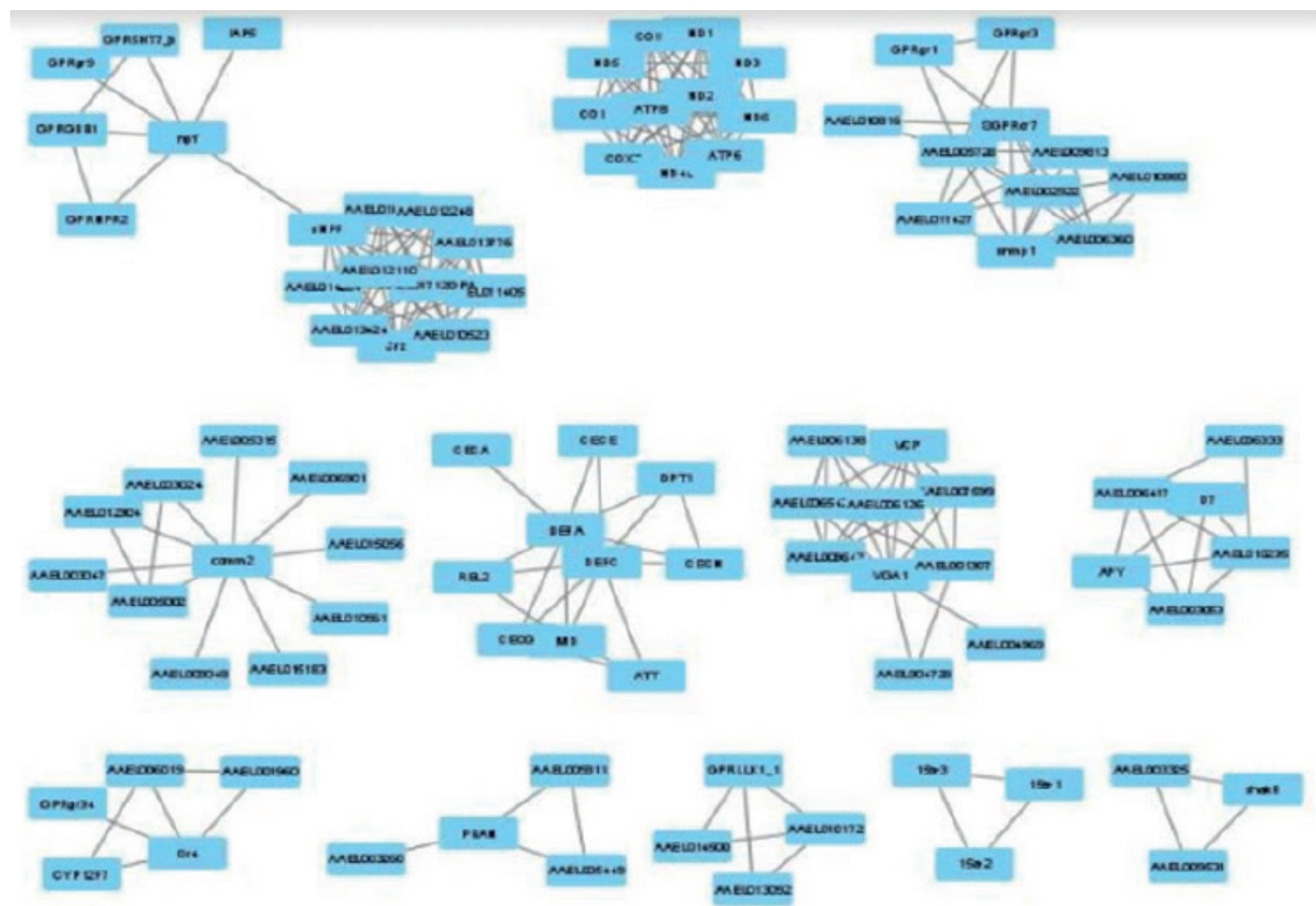

obes

Figure 2. Merged PPI network having 96 nodes, 243 edges in Cytoscape.
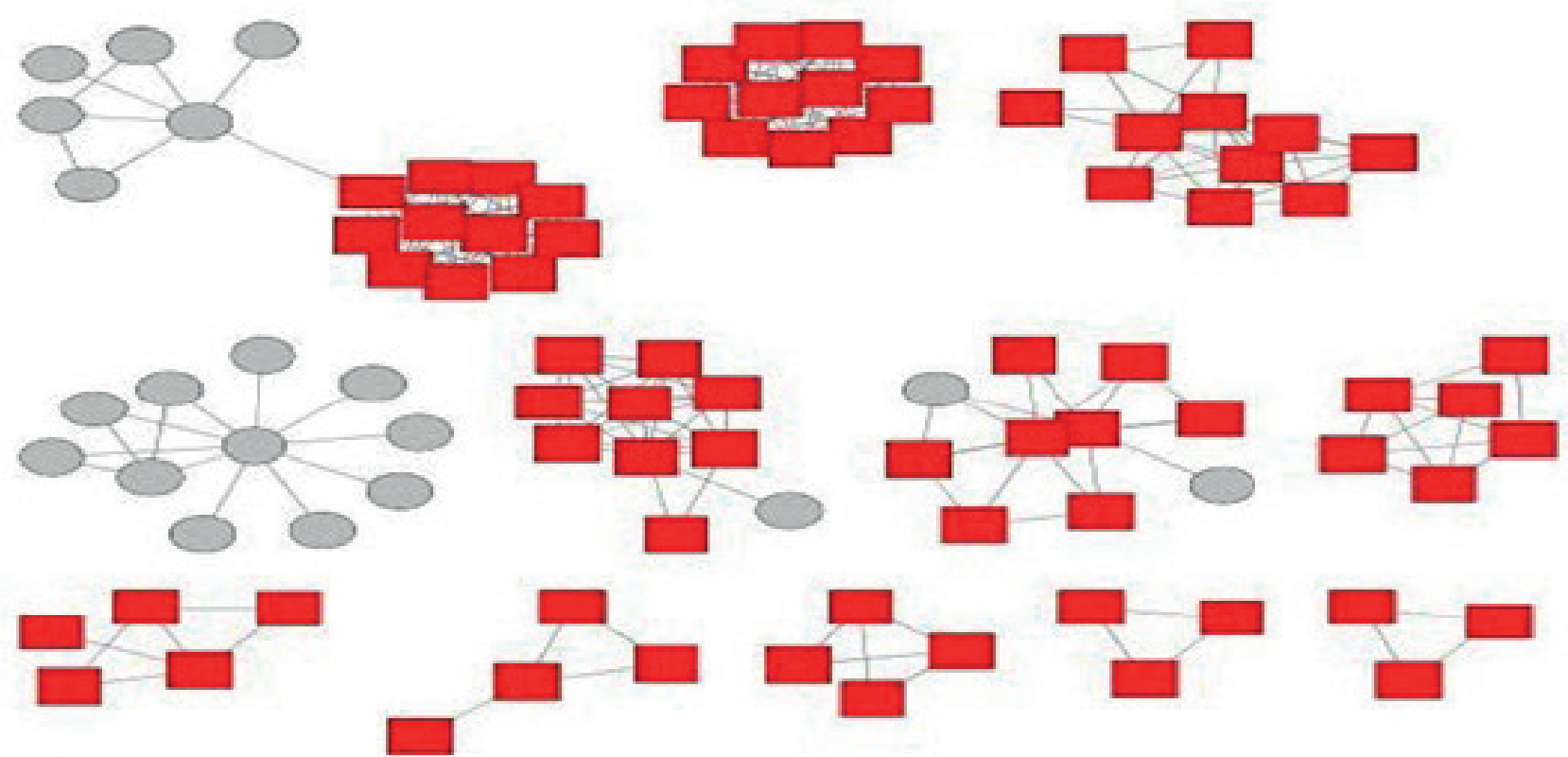

Figure 3. ClusterONE results were obtained by analyzing the interaction network. 
PPI network (Figure 2) to identify the "Hubs" which represent the highly connected nodes. A hub is more likely to be essential because it has more interactions and, therefore, a higher likelihood to engage in an essential interaction ${ }^{31}$. The network obtained showed genes/proteins color-coded with highly significant genes in red (31 genes), orange and least significant in yellow (Figure 5).

The proteins present in the three top-ranked clusters obtained from ClusterONE and MCODE were identical, as were the 31 top-ranked genes obtained from CytoHubba, thereby validating our results obtained from different Cytoscape plugins that use different methods to identify hub proteins that can further be explored to act as putative target proteins for vector control (Table 4).

\section{Data mining of shortlisted proteins}

UniProtKB was used to retrieve information, such as target name, associated metabolic pathway, length, molecular weight, subcellular location etc., of the identified 31 essential genes of Aedes genus that code for non-homologous human proteins. Thereafter, a rigorous manual search in PDB was done to identify any structural homologs amongst the proteins of Aedes genus with its human host as unintended binding of the drug/vaccine, designed for Aedes to the structurally similar proteins of the human host could have devastating effects in the hosts, since structurally similar and related proteins may have similar binding sites with capabilities to recognize chemically similar ligands ${ }^{32}$.

Out of 31 proteins of Aedes only 9 proteins did not have any structural homology with humans in PDB. These 09 proteins shortlisted three other proteins, namely BRUS_AEDAE,
HPEP_AEDAE (that had no entries in STRING database), and ALL3_AEDAE (salivary gland allergen reported to have binary interactions with 2 human proteins, namely COA1 and CO2A3) were analyzed for their biological function in UniProt. Therefore, 12 proteins were shortlisted as novel vector-control target proteins of $A$. aegypti that had no human homologs and could effectively act as potential drug and vaccine targets (Table 5) to prevent the outbreak of VBDs by controlling the vector population of Aedes.

\section{Literature mining of critical vector-control target proteins of $A$. aegypti}

In the present study to identify critical vector-control target proteins for Aedes genus that can be used as potential targets for vaccine and drug development, we identified 12 proteins, out of which 5 proteins are neuropeptides. Two uncharacterized proteins, AAELO13776_AEDAE and AAEL013424_AEDAE, need to be further solved in structure and function to predict their importance in vector metabolic activities and survival.

Neuropeptides are one of the most diverse classes of signaling molecules and play a significant role in regulating a wide range of physiological processes in Aedes mosquitoes such as host-seeking behavior, blood-feeding, molting sex-specific processes, including reproduction ${ }^{33}$. Regulatory peptides act as neurochemicals, and hormones govern these processes in mosquitoes. These peptides are processed, stored, and released within the nervous system as neurotransmitters and from the midgut endocrine system and neurosecretory cells as circulating hormones. They exert their action by binding to membrane receptors, most often to G-protein coupled re-

\begin{tabular}{|l|l|l|l|l|l|l|l|}
\hline $\begin{array}{l}\text { Sr. } \\
\text { No. }\end{array}$ & Clusters & $\begin{array}{l}\text { No. of } \\
\text { Nodes }\end{array}$ & P-value & $\begin{array}{c}\text { Sr. } \\
\text { No. }\end{array}$ & Clusters & $\begin{array}{l}\text { No. of } \\
\text { Nodes }\end{array}$ & \multicolumn{1}{|c|}{ P-value } \\
\hline 1 & 11 & $2.705 \mathrm{E}-6$ & 7 & & & \\
\hline
\end{tabular}

Table 2. Eleven clusters having significant P-value $(<0.05)$ obtained from ClusterONE. 

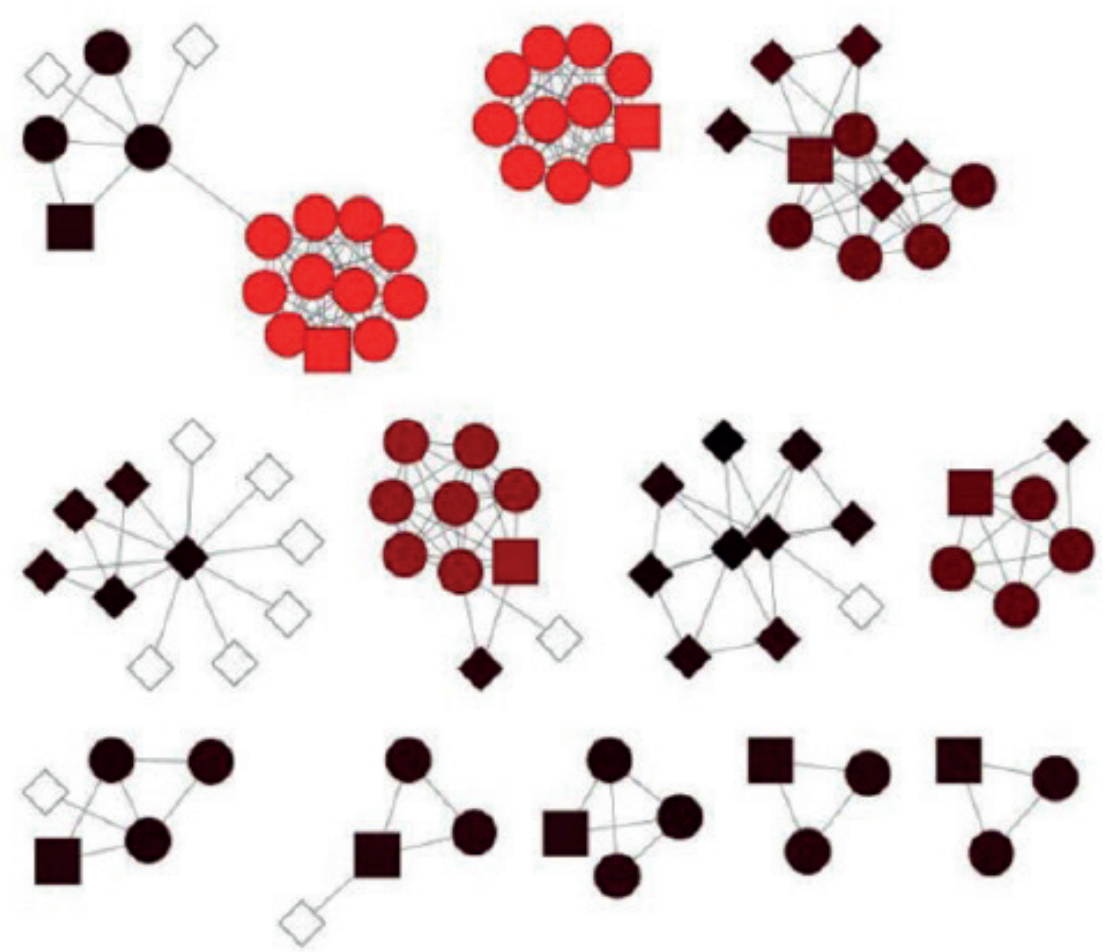

Figure 4. MCODE results obtained by analyzing the PPI network.

\begin{tabular}{|c|c|c|c|c|c|c|c|}
\hline No. & Clusters & Nodes & Edges & S. No. & Clusters & Nodes & Edges \\
\hline 1 & & 11 & 55 & 7 & & 4 & 5 \\
\hline 2 & & 11 & 55 & 8 & & 4 & 5 \\
\hline 3 & & 8 & 27 & 9 & & 3 & 3 \\
\hline 4 & & 5 & 10 & 10 & & 3 & 3 \\
\hline 5 & & 6 & 12 & 11 & & 3 & 3 \\
\hline 6 & & 4 & 5 & & & & \\
\hline
\end{tabular}

Table 3. Eleven clusters were obtained from the interaction network. 

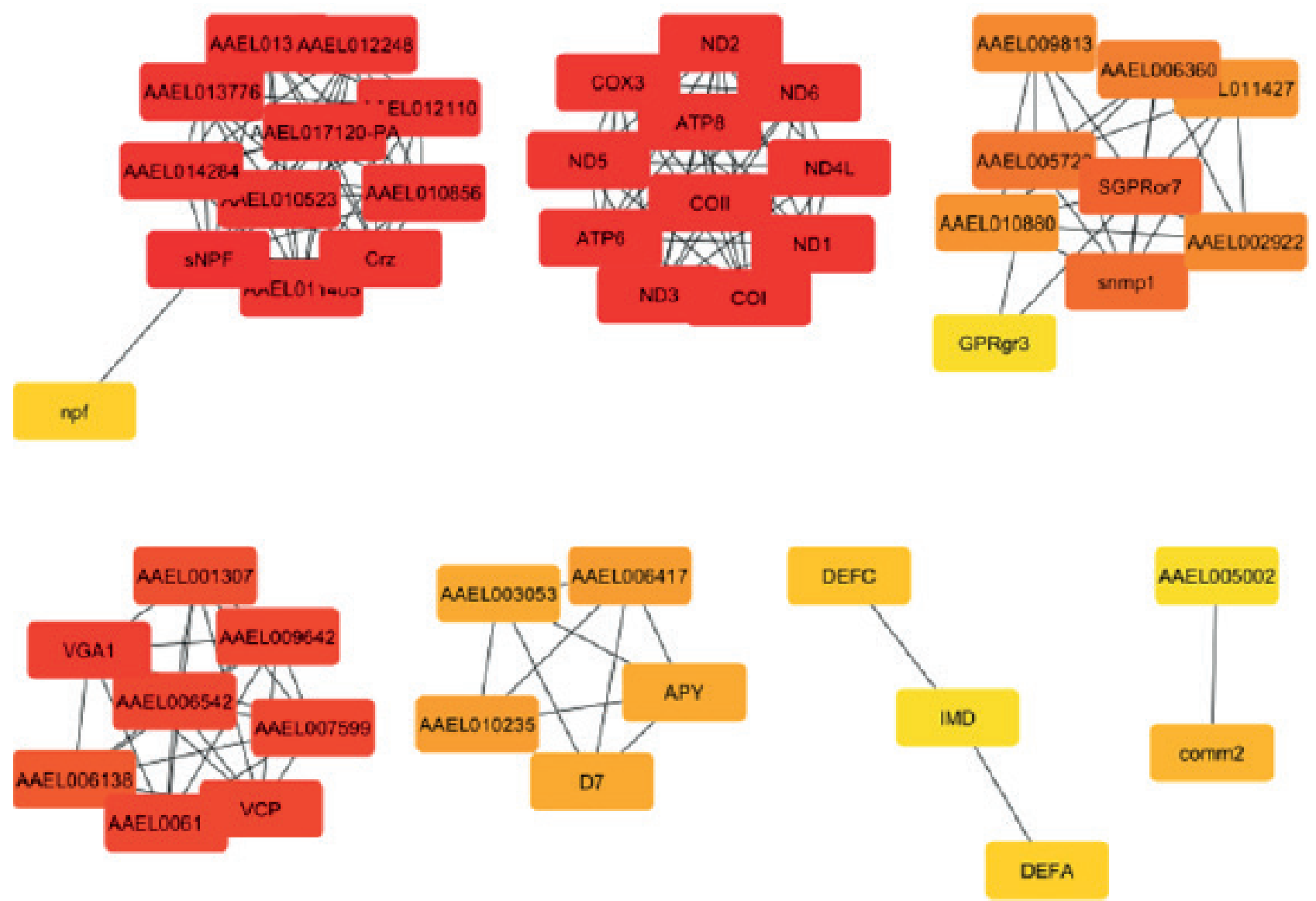

Figure 5. Graphical view of ranked hub nodes obtained from Cytoscape plugin Cytohubba with color-coding highly essential (Red) - less essential (Yellow).

ceptors (GPCRs) and, to a lesser extent, to receptor tyrosine kinases $^{34}$.

Host seeking is a fundamental aspect of mosquito and vertebrate host interactions, and the process is reported to be regulated by head peptide (HPEP_AEDAE), a neuropeptide encoded by gene HP-1 in blood-fed female A. aegypti, and also other sex-specific processes as it was found to be expressed in larvae and males. Ingestion of a blood meal has been reported to trigger egg maturation in A. aegypti, and after that, females show a marked decrease in responses to host emanations that initially stimulated host-seeking behavior ${ }^{35}$.

Short neuropeptide F (SNPF_AEDAE), a member of the neuropeptide $F / Y$ superfamily, was isolated from adult $A$. aegypti, and NPF-like immunostaining was observed in the brain and midgut of adults and larvae. The reported fluctuations in the concentrations of sNPF reflect changes in the regulatory role of this peptide in digestive processes and the reproductive cycle with a sudden drop once a blood meal was imbibed ${ }^{28}$.

Proteins such as odorant receptors (ORs) play critical roles in olfactory behaviors, including a co-receptor, designated ORCO_AEDAE, expressed in most olfactory sensory neurons in both adults and larvae, and is highly conserved among the order Diptera to which the Aedes genus belongs. It plays a crucial role in forming a ligand-gated ion channel to generate signals to induce behavioral responses, including host-seeking and host selection ${ }^{36}$.

The arthropod saliva contains proteins delivered into the bite wound that can modulate the host's hemostatic and immune responses to facilitate blood meal intake and pathogen transmission. Aegyptin (ALL3_AEDAE), an abundantly expressed salivary protein of $A$. aegypti, is a collagen-binding protein that inhibits platelet aggregation and adhesion, thereby playing a significant and nonredundant role in successful feeding ${ }^{29,37}$. It has been reported to have interactions with the human collagen alpha-1 (I) and (III) chains i.e., COA1_HUMAN \& CO2A3_HUMAN, respectively, thereby helping to transmit the pathogen in the vector saliva to the human host.

Gonadotropin-releasing hormone-related peptide Corazonin (CRZ) has been shown to have roles in pigmentation, ecdysis or act as a cardio stimulatory factor while its receptor-CRZR is expressed in the primary sex organs of adult male and female mosquitoes. This indicates a potential physiological role of CORZ_AEDAE in regulating reproductive biology in A. aegypti37.

The gene encoding vitellogenin $(\mathrm{Vg})$, a significant yolk protein precursor (YPP) in most oviparous animals, is expressed in extra ovarian tissues in a sex-, tissue- and stage-specific manner. Steroid hormones play a critical role in the regulation of $\mathrm{Vg}$ genes. In egg-laying vertebrates, estrogen triggers the transcription of the $\mathrm{Vg}^{38}$. Before blood-feeding, ovary maturation remains at a previtellogenic state of arrest, and the production of vitellogenin (VIT1_AEDAE), the major yolk protein precursor, by the fat body is tightly repressed. After a blood meal, multiple regulatory factors act synergistically to trigger vitellogenin protein synthesis in the fat body. The vitellogenin proteins are then released into the hemolymph and taken up by the developing oocytes via receptor-mediated endocytosis. Therefore, stringent control of the expression of the $\mathrm{Vg}$ gene in mosquitoes is tied to the availability of a blood meal ${ }^{30}$.

Two other unreviewed proteins shortlisted in the present study, namely Q177I5_AEDAE and Q177I2_AEDAE have reported lipid transporter activity, and on the investigation of their 


\begin{tabular}{|c|c|c|c|c|c|}
\hline \multicolumn{2}{|c|}{ ClusterONE } & \multicolumn{2}{|c|}{ CytoHubba } & \multirow{2}{*}{\multicolumn{2}{|c|}{ MCODE }} \\
\hline & & RANK & Protein & & \\
\hline \multirow{11}{*}{ 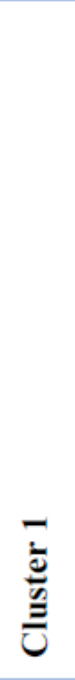 } & ND1 & 1 & sNPF & ND1 & \multirow{11}{*}{ 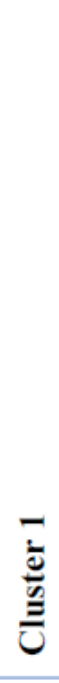 } \\
\hline & ND3 & 2 & AAEL017120-PA & ND3 & \\
\hline & ND5 & 2 & AAEL010856 & ND2 & \\
\hline & ND6 & 2 & AAEL014284 & ND6 & \\
\hline & ATP6 & 2 & AAEL 012248 & ATP6 & \\
\hline & ND4L & 2 & AAEL012110 & ND4L & \\
\hline & $\mathrm{COX} 3$ & 2 & AAEL013424 & $\mathrm{COX} 3$ & \\
\hline & $\mathrm{COI}$ & 2 & AAEL 010523 & $\mathrm{COi}$ & \\
\hline & ATP8 & 2 & AAEL013776 & ND5 & \\
\hline & ND2 & 2 & AAEL011405 & COii & \\
\hline & COII & 2 & $\mathrm{Crz}$ & ATP8 & \\
\hline & & 2 & COii & & \\
\hline \multirow{11}{*}{ 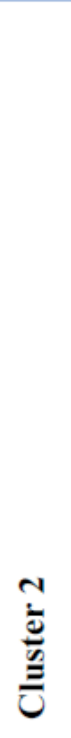 } & AAEL 012248 & 2 & $\mathrm{COi}$ & AAEL 010856 & \multirow{11}{*}{ 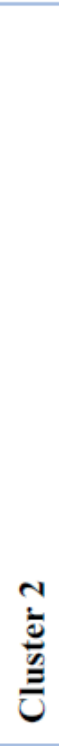 } \\
\hline & AAEL 012110 & 2 & ND1 & AAEL 012248 & \\
\hline & AAEL 014284 & 2 & ND2 & AAEL 013776 & \\
\hline & AAEL 010856 & 2 & $\mathrm{COX} 3$ & AAEL011405 & \\
\hline & AAEL011405 & 2 & ND5 & AAEL 010523 & \\
\hline & $\mathrm{Crz}$ & 2 & ND3 & AAEL017120-PA & \\
\hline & AAEL 013776 & 2 & ND4L & AAEL012110 & \\
\hline & AAEL 010523 & 2 & ATP6 & AAEL013424 & \\
\hline & $\begin{array}{l}\text { AAEL017120-P } \\
\text { A }\end{array}$ & 2 & ATP8 & AAEL014284 & \\
\hline & sNPF & 2 & ND6 & $\mathrm{Crz}$ & \\
\hline & AAEL013424 & 23 & VGA1 & sNPF & \\
\hline & & 24 & AAEL007599 & & \\
\hline \multirow{11}{*}{ 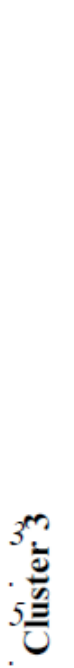 } & AAEL010816 & 24 & $\mathrm{VCP}$ & $\mathrm{VCP}$ & \multirow{11}{*}{ 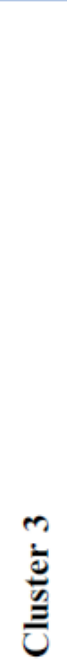 } \\
\hline & SGPRor7 & 24 & AAEL009642 & VGA1 & \\
\hline & GPRgr1 & 24 & AAEL006542 & AAEL007599 & \\
\hline & AAEL 011427 & 24 & AAEL006126 & AAEL001307 & \\
\hline & AAEL002922 & 29 & AAEL001307 & AAEL006126 & \\
\hline & AAEL006360 & 30 & AAEL006138 & AAEL009642 & \\
\hline & Snmp1 & 31 & SGPRor7 & AAEL006542 & \\
\hline & AAEL009813 & & & AAEL006138 & \\
\hline & AAEL005278 & & & & \\
\hline & AAEL 010880 & & & & \\
\hline & GPRgr3 & & & & \\
\hline
\end{tabular}

Table 4. Top interacting Genes retrieved from Cytoscape plugins. 


\begin{tabular}{|c|c|c|c|c|c|c|}
\hline S. No. & Entry name & $\begin{array}{l}\text { Non homologous } \\
\text { protein target } \\
\text { name }\end{array}$ & 竞 & $\begin{array}{l}\text { Associated metabolic pathway } \\
\text { (Uniprot/EggNOG) }\end{array}$ & 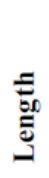 & 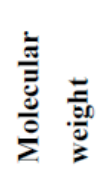 \\
\hline 1. & ATP8_AEDAE & $\begin{array}{l}\text { ATP synthase } \\
\text { protein } 8\end{array}$ & B0FWC9 & - Energy production and conversion & $\hat{n}$ & $\tilde{m}$ \\
\hline 2. & $\begin{array}{l}\text { AAEL013776_A } \\
\text { EDAE }\end{array}$ & $\begin{array}{l}\text { Uncharacterized } \\
\text { protein }\end{array}$ & Q16I67 & $\begin{array}{l}\text { - Translation } \\
\text { - ribosomal structure and biogenesis }\end{array}$ & $\stackrel{\text { q }}{=}$ & $\underset{\mathfrak{J}}{\stackrel{N}{\sim}}$ \\
\hline 3. & $\begin{array}{l}\text { AAEL013424_A } \\
\text { EDAE }\end{array}$ & $\begin{array}{l}\text { Uncharacterized } \\
\text { protein }\end{array}$ & Q16J76 & - Function unknown & $\stackrel{m}{I}$ & $\stackrel{+}{\stackrel{0}{2}}$ \\
\hline 4. & CORZ_AEDAE & Pro-corazonin & Q17AN4 & $\begin{array}{l}\text { - Corazonin receptor binding } \\
\text { - Neuropeptide signaling } \\
\text { - Positive regulation of heart contraction }\end{array}$ & $\hat{n}$ & ڤิ \\
\hline 5. & SNPF_AEDAE & $\begin{array}{l}\text { Short neuropeptide } \\
\text { F }\end{array}$ & A0SIX6 & $\begin{array}{l}\text { - Neuropeptide signaling } \\
\text { - Regulation of multicellular organism } \\
\text { growth } \\
\text { - Regulation of response to food }\end{array}$ & $\stackrel{n}{\sim}$ & ڤ̂ర \\
\hline 6. & VIT1_AEDAE & Vitellogenin-A1 & Q16927 & $\begin{array}{l}\text { - Lipid transporter activity } \\
\text { - Nutrient reservoir activity }\end{array}$ & $\frac{\text { ga }}{2}$ & 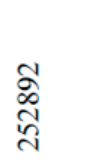 \\
\hline 7. & Q177I5_AEDAE & AAEL006126-PA & Q177I5 & - Lipid transporter activity & $\stackrel{\infty}{\stackrel{\infty}{0}}$ & 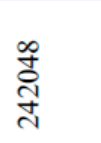 \\
\hline 8. & Q177I2_AEDAE & AAEL006138-PA & Q177I2 & - Lipid transporter activity & $\stackrel{\stackrel{\sim}{ \pm}}{\mathrm{N}}$ & $\underset{\substack{+\stackrel{2}{+}}}{\stackrel{2}{2}}$ \\
\hline 9. & BURS_AEDAE & Bursicon & P85316 & $\begin{array}{l}\text { - Cuticle pigmentation } \\
\text { - Chitin-based cuticle sclerotization } \\
\text { - Signal transduction } \\
\text { - Hormone activity }\end{array}$ & $\underline{\underline{0}}$ & $\stackrel{\frac{2}{2}}{\underline{2}}$ \\
\hline 10. & ALL3_AEDAE & $\begin{array}{l}30 \mathrm{kDa} \text { salivary } \\
\text { gland allergen } \\
\text { Aed a } 3\end{array}$ & $\begin{array}{l}\text { AAEL010 } \\
235\end{array}$ & $\begin{array}{l}\text { - Causes an allergic reaction in humans } \\
\text { by interaction with COA1_HUMAN \& } \\
\text { CO2A3_HUMAN }\end{array}$ & $\stackrel{\text { }}{\wedge}$ & $\frac{n}{2}$ \\
\hline 11. & HPEP_AEDAE & Head peptide & Q9GQV7 & $\begin{array}{l}\text { - Neuropeptide signaling pathway } \\
\text { - Negative regulation of host-seeking } \\
\text { behavior }\end{array}$ & $\stackrel{\infty}{\simeq}$ & 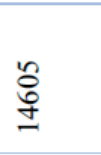 \\
\hline 12. & ORCO_AEDAE & $\begin{array}{l}\text { Odorant receptor } \\
\text { coreceptor }\end{array}$ & Q178U6 & $\begin{array}{l}\text { - Detection of chemical stimulus involved } \\
\text { in sensory perception of smell } \\
\text { - Signal transduction } \\
\text { - Eating behavior }\end{array}$ & $\stackrel{\infty}{\stackrel{\infty}{f}}$ & $\underset{\text { N }}{\stackrel{N}{N}}$ \\
\hline
\end{tabular}

Table 5. Non-homologous proteins of $A$. aegypti with reference to humans as potential drug and vaccine targets from unique pathways. 
families and domains using InterPro, Pfam and Prosite databases, they were found to be related to vitellogenin, thereby indicating that they might also be involved in the process of oogenesis.

Bursicon is a neuropeptide hormone consisting of two subunits, burs $\mathrm{a}$ and burs $\beta$, members of the cystine-knot protein (CKP) family that usually form dimers (homo- or heterodimers) and are responsible for certain biological functions by binding to specific receptors. Zhang et al.(2017) report that the biological significance of bursicon extends beyond its classical developmental roles in cuticle tanning and wing expansion to mediating prophylactic immunity during molting periods. Bursicon (BURS_AEDAE) homodimers were shown to induce expression of AMP genes via Relish2 in A. aegypti as prophylactic immunity to protect mosquitoes during the vulnerable stages of each molt ${ }^{39}$.

The $F_{1}-F_{0}$ ATP synthase is a large multi-subunit enzyme that uses the proton gradient generated by the respiratory chain to synthesize ATP. $F_{1}$-ATP synthase region is composed of five different subunits $(a, \beta, \gamma, \delta, \varepsilon)$ assembled as a complex of three alternating $a$ and $\beta$ subunits together with a single unit of each of the other three subunits ${ }^{40}$. It was initially believed that $F_{1}-F_{0}$ ATP synthase was strictly confined to the mitochondrial inner membrane. But for a wide variety of normal cells, including endothelial cells, adipocytes, keratinocytes, and hepatic cells, active ATP synthase activity on the cell surface has been documented ${ }^{41}$. Fongsaran et al.(2014) reported that ATPS $\beta$ plays a role in Chikungunya virus (CHIKV) infection of mosquito cells ${ }^{41}$, and there is evidence showing that the same protein is involved in the internalization of white spot syndrome virus (WSSV) to shrimp cells ${ }^{42}$, and possibly binding of dengue virus to insect cells ${ }^{43}$, suggests that this is a conserved virus-arthropod interaction. Therefore, by targeting ATP8_AE$\mathrm{DAE}$, the interaction of the pathogens and the mosquito vector can be disrupted and thereby, transmission to the human host may be effectively prevented.

Furthermore, targeting proteins involved in various physiological processes such as reproductive (CORZ_AEDAE and VIT1_AEDAE), immune (BURS_AEDAE) and metabolic activities (ATP8_AEDAE) can affect the survival of the vectors and thereby contain the spread of VBDs by controlling vector population.

Concomitantly, blood-feeding on humans also propagates disease transmission because the mosquito ingests the pathogen from an infected human and transmits it to new hosts during subsequent blood meals. The concentration of Head peptide and SNPF are higher in mosquitoes before blood feeding i.e., during the host-seeking phase and following blood meal, their concentrations fall ${ }^{44,45}$. Therefore, Head peptide (HPEP_AEDAE), sNPF (SNPF_AEDAE), ORCO (ORCO_AEDAE) and ALL3 (ALL3_AEDAE) may also prove useful targets for blocking host-seeking and biting behavior, thereby preventing the transmission of pathogens from vector to the human host and thus constitute an alternative strategy to curb the disease.

\section{Conclusions}

The proteins identified in the present study can develop novel strategies to target critical vector components to successfully block the development of the vector or pathogen/ parasite in its vector.

\section{Funding}

The authors would like to acknowledge the fun- ding received under grant no. S\&T\&RE/Rp/147(19-20) San/10/2019/1703-1710 from Department of Science and Technology and Renewable Energy, U.T, Chandigarh, India under the short term research project scheme along with Panjab University Chandigarh for providing the infrastructure for the research work.

\section{Informed Consent Statement}

Not applicable.

\section{Acknowledgments}

The authors would like to acknowledge their parent institute, Panjab University Chandigarh, for providing the infrastructure for the work.

\section{Conflicts of Interest}

The authors declare no conflict of interest.

\section{Bibliographic references}

1. WHO. Vector borne diseases Report available at https://www. who.int/news-room/fact-sheets/detail/vector-borne-diseases.

2. Roiz D, Wilson AL, Scott TW, Fonseca DM, Jourdain F, Müller P, Velayudhan R, Corbel V. Integrated Aedes management for the control of Aedes-borne diseases. PLoS neglected tropical diseases. 2018 Dec 6;12(12):e0006845.

3. Mayer SV, Tesh RB, Vasilakis N. The emergence of arthropod-borne viral diseases: A global prospective on dengue, chikungunya and zika fevers. Acta tropica. 2017 Feb 1;166:155-63.

4. Kraemer MU, Reiner RC, Brady OJ, Messina JP, Gilbert M, Pigott DM, Yi D, Johnson K, Earl L, Marczak LB, Shirude S. Past and future spread of the arbovirus vectors Aedes aegypti and Aedes albopictus. Nature microbiology. 2019 May;4(5):854-63.

5. Weaver SC, Reisen WK. Present and future arboviral threats. Antiviral research. 2010 Feb 1;85(2):328-45.

6. Barrera R, Amador M, Diaz A, Smith J, Munoz区Jordan JL, Rosario Y. Unusual productivity of Aedes aegypti in septic tanks and its implications for dengue control. Medical and veterinary entomology. 2008 Mar;22(1):62-9.

7. Invasive Species Specialist Group. Global Invasive Species Database - Aedes albopictus 2009.

8. Eritja R, Escosa R, Lucientes J, Marques E, Roiz D, Ruiz S. Worldwide invasion of vector mosquitoes: present European distribution and challenges for Spain. Biological invasions. 2005 Mar;7(1):8797.

9. Turell MJ, Dohm DJ, Sardelis MR, O'guinn ML, Andreadis TG, Blow JA. An update on the potential of North American mosquitoes (Diptera: Culicidae) to transmit West Nile virus. Journal of medical entomology. 2005 Jan 1;42(1):57-62.

10. Tolle MA. Mosquito-borne diseases. Current problems in pediatric and adolescent health care. 2009 Apr 1;39(4):97-140.

11. UniProt Consortium. UniProt: a worldwide hub of protein knowledge. Nucleic acids research. 2019 Jan 8;47(D1):D506-15.

12. Huerta-Cepas J, Szklarczyk D, Heller D, Hernández-Plaza A, Forslund SK, Cook H, Mende DR, Letunic I, Rattei T, Jensen LJ, von Mering C. eggNOG 5.0: a hierarchical, functionally and phylogenetically annotated orthology resource based on 5090 organisms and 2502 viruses. Nucleic acids research. 2019 Jan 8:47(D1):D309-14.

13. Madden T. The BLAST sequence analysis tool. InThe NCBI Handbook [Internet]. 2nd edition 2013 Mar 15. National Center for Biotechnology Information (US).

14. Kanehisa M. The KEGG database In: Novartis Foundation Symposium.

15. Yu CS, Cheng CW, Su WC, Chang KC, Huang SW, Hwang JK, Lu CH. CELLO2GO: a web server for protein subCELlular LOcalization prediction with functional gene ontology annotation. PloS one. 2014 Jun 9;9(6):e99368. 
16. Lin WZ, Fang JA, Xiao X, Chou KC. iLoc-Animal: a multi-label learning classifier for predicting subcellular localization of animal proteins. Molecular BioSystems. 2013;9(4):634-44.

17. Briesemeister S, Blum T, Brady S, Lam Y, Kohlbacher O, Shatkay H. SherLoc2: a high-accuracy hybrid method for predicting subcellular localization of proteins. Journal of proteome research. 2009 Nov 6;8(11):5363-6.

18. Fu SC, Imai K, Horton P. Prediction of leucine-rich nuclear export signal containing proteins with NESsential. Nucleic acids research. 2011 Sep 1;39(16):e111-.

19. Mering CV, Huynen M, Jaeggi D, Schmidt S, Bork P, Snel B. STRING: a database of predicted functional associations between proteins. Nucleic acids research. 2003 Jan 1;31(1):258-61.

20. Nepusz T, Yu H, Paccanaro A. Detecting overlapping protein complexes in protein-protein interaction networks. Nature methods. 2012 May;9(5):471.

21. Chin $\mathrm{CH}$, Chen $\mathrm{SH}$, Wu HH, Ho CW, Ko MT, Lin CY. cytoHubba: identifying hub objects and sub-networks from complex interactome. BMC systems biology. 2014 Dec;8(4):1-7.

22. Bader GD, Hogue CW. An automated method for finding molecular complexes in large protein interaction networks. BMC bioinformatics. 2003 Dec; 4(1):1-27.

23. Singh S, Malik BK, Sharma DK. Metabolic pathway analysis of S. pneumoniae: an in silico approach towards drug-design. Journal of bioinformatics and computational biology. 2007 Feb;5(01):13553.

24.Gabaldón T, Koonin EV. Functional and evolutionary implications of gene orthology. Nature Reviews Genetics. 2013 May;14(5):3606.

25. Merkling SH, van Rij RP. Beyond RNAi: antiviral defense strategies in Drosophila and mosquito. Journal of insect physiology. 2013 Feb 1;59(2):159-70.

26. Oktarianti R, Senjarini K, Hayano T, Fatchiyah F. Proteomic analysis of immunogenic proteins from salivary glands of Aedes aegypti. Journal of infection and public health. 2015 Nov 1;8(6):575-82.

27. Chagas AC, Ramirez JL, Jasinskiene N, James AA, Ribeiro JM, Marinotti O, Calvo E. Collagen-binding protein, Aegyptin, regulates probing time and blood feeding success in the dengue vector mosquito, Aedes aegypti. Proceedings of the National Academy of Sciences. 2014 May 13;111(19):6946-51.

28. Stanek DM, Pohl J, Crim JW, Brown MR. Neuropeptide F and its expression in the yellow fever mosquito, Aedes aegypti. Peptides. 2002 Aug 1;23(8):1367-78.

29. Engelmann F. Insect vitellogenin: identification, biosynthesis, and role in vitellogenesis. InAdvances in Insect Physiology 1979 Jan 1 (Vol. 14, pp. 49-108). Academic Press.

30.Martı 囚n D, Wang SF, Raikhel AS. The vitellogenin gene of the mosquito Aedes aegypti is a direct target of ecdysteroid receptor. Molecular and cellular endocrinology. 2001 Feb 22;173(1-2):7586.

31. He X, Zhang J. Why do hubs tend to be essential in protein networks? PLoS Genet. 2006 Jun 2;2(6):e88.
32. Govindaraj RG, Brylinski M. Comparative assessment of strategies to identify similar ligand-binding pockets in proteins. BMC bioinformatics. 2018 Dec;19(1):1-7.

33. Predel R, Neupert S, Garczynski SF, Crim JW, Brown MR, Russell WK, Kahnt J, Russell DH, Nachman RJ. Neuropeptidomics of the mosquito Aedes aegypti. Journal of proteome research. 2010 Apr 5;9(4):2006-15.

34. Riehle MA, Garczynski SF, Crim JW, Hill CA, Brown MR. Neuropeptides and peptide hormones in Anopheles gambiae. Science. 2002 Oct 4;298(5591):172-5.

35. Stracker TH, Thompson S, Grossman GL, Riehle MA, Brown MR. Characterization of the AeaHP gene and its expression in the mosquito Aedes aegypti (Diptera: Culicidae). Journal of medical entomology. 2002 Mar 1;39(2):331-42.

36. Liu H, Liu T, Xie L, Wang X, Deng Y, Chen CH, James AA, Chen XG. Functional analysis of Orco and odorant receptors in odor recognition in Aedes albopictus. Parasites \& vectors. 2016 Dec;9(1):1-0.

37. Oryan A, Wahedi A, Paluzzi JP. Functional characterization and quantitative expression analysis of two $\mathrm{GnRH}$-related peptide receptors in the mosquito, Aedes aegypti. Biochemical and biophysical research communications. 2018 Mar 4;497(2):550-7.

38. Wetekam W, Mullinix KP, Deeley RG, Kronenberg HM, Eldridge JD, Meyers M, Goldberger RF. Effect of estrogen on gene expression: purification of vitellogenin messenger RNA. Proceedings of the National Academy of Sciences. 1975 Sep 1;72(9):3364-8.

39.Zhang H, Dong S, Chen X, Stanley D, Beerntsen B, Feng Q, Song Q. Relish2 mediates bursicon homodimer-induced prophylactic immunity in the mosquito Aedes aegypti. Scientific reports. 2017 Feb 22;7(1):1-9.

40.Leyva JA, Bianchet MA, Amzel LM. Understanding ATP synthesis: structure and mechanism of the F1-ATPase. Molecular membrane biology. 2003 Jan 1;20(1):27-33.

41. Fongsaran C, Jirakanwisal K, Kuadkitkan A, Wikan N, Wintachai P, Thepparit C, Ubol S, Phaonakrop N, Roytrakul S, Smith DR. Involvement of ATP synthase $\otimes$ subunit in chikungunya virus entry into insect cells. Archives of virology. 2014 Dec;159(12):3353-64.

42.Paingankar MS, Gokhale MD, Deobagkar DN. Dengue-2-virus-interacting polypeptides involved in mosquito cell infection. Archives of virology. 2010 Sep 1;155(9):1453-61.

43.Zhan W, Wang X, Chi Y, Tang X. The VP37-binding protein F1ATP synthase $\otimes$ subunit involved in WSSV infection in shrimp Litopenaeus vannamei. Fish \& shellfish immunology. 2013 Jan 1;34(1):228-35.

44.Christ P, Reifenrath A, Kahnt J, Hauser F, Hill SR, Schachtner $J$, Ignell R. Feeding-induced changes in allatostatin-A and short neuropeptide $F$ in the antennal lobes affect odor-mediated host seeking in the yellow fever mosquito, Aedes aegypti. PloS one. 2017 Nov 22;12(11):e0188243.

45.Duvall, L. B., Ramos-Espiritu, L., Barsoum, K. E., Glickman, J. F., \& Vosshall, L. B. (2019). Small-molecule agonists of Ae. aegypti neuropeptide $Y$ receptor block mosquito biting. Cell, 176(4), 687701. 